\title{
Roles of ATR1 paralogs YMR279c and YOR378w in boron stress tolerance
}

\author{
Gonensin Ozan Bozdag, Irem Uluisik, Gulce Sila Gulculer, Huseyin C. Karakaya, Ahmet Koc* \\ Izmir Institute of Technology, Department of Molecular Biology and Genetics, 35430 Urla, Izmir, Turkey
}

\section{A R T I C L E I N F O}

\section{Article history:}

Received 6 May 2011

Available online 20 May 2011

\section{Keywords:}

Boron

Boric acid

Yeast

Stress response

ATR1

Ymr279c

\begin{abstract}
A B S T R A C T
Boron is a necessary nutrient for plants and animals, however excess of it causes toxicity. Previously, Atr1 and Arabidopsis Bor1 homolog were identified as the boron efflux pump in yeast, which lower the cytosolic boron concentration and help cells to survive in the presence of toxic amount of boron. In this study, we analyzed ATR1 paralogs, YMR279c and YOR378w, to understand whether they participate in boron stress tolerance in yeast. Even though these genes share homology with ATR1, neither their deletion rendered cells boron sensitive nor their expression was significantly upregulated by boron treatment. However, expression of YMR279, but not YOR378w, from the constitutive GAPDH promoter on a high copy plasmid provided remarkable boron resistance by decreasing intracellular boron levels. Thus our results suggest the presence of a third boron exporter, YMR279c, which functions similar to ATR1 and provides boron resistance in yeast.
\end{abstract}

(c) 2011 Elsevier Inc. All rights reserved.

\section{Introduction}

Boron is an essential micronutrient for various species [1-4]. The essentiality of boron is known almost for 90 years for plants [5]. In plants, boron act as a cross-linker of rhamnogalacturonanII molecule [6] within the cell wall and maintains stability of the plant cell structure, and its deficiency has been linked to growth problems in Arabidopsis thaliana [4].

A. thaliana Bor1 is the first identified boron transporter, functions as an efflux-pump and essential when limited amount of boron is available for growth [7]. Yeast Bor1 has been identified as a boron exporter having role in boron tolerance [8]. In a recent study, Atr1 was suggested as the main boron exporter in yeast [9]. Atr1 deficient cells were boron sensitive and accumulated higher level of intracellular boron, whereas ATR1 overexpressing cells were resistant to toxic amount of boron application and contained less boron. ATR1 transcription was induced several fold by boron treatment [9].

Yeast ATR1 belongs to the DHA2 family of drug: $\mathrm{H}^{+}$antiporters with 10 members in the budding yeast, and this transporter family is conserved among yeast species such as Kluyveromyces lactis, Kluyveromyces waltii, Candida glabrata, Ashbya gossypii [10]. Seven of DHA2 family members including YMR279C and YOR378W have not yet been characterized [11]. Since YMR279C and YOR378W are the two closest paralogs of ATR1, we speculated that they may play a role in boron stress response. To test this idea, both genes were cloned and overexpressed in yeast. Expression of

\footnotetext{
* Corresponding author.

E-mail address: ahmetkoc@iyte.edu.tr (A. Koc).
}

YMR279C, but not YOR378W, provided boron resistance by decreasing intracellular boron level.

\section{Materials and methods}

\subsection{Yeast growth and media}

WT strain BY4741 (MATa his3 leu2 met15 ura3) and its isogenic atr1 $\Delta, y m r 279 c \Delta$, and yor378w $\Delta$ deletion mutants were obtained from the yeast deletion library (Invitrogen). YPD (2\% glucose, $2 \%$ peptone, $1 \%$ yeast extract, and $2 \%$ agar for solid media) and YNB media $(0,67 \%$ yeast nitrogen base without amino acids, $2 \%$ glucose, $2 \%$ agar for solid media) were used for cell growth. For spotting assays, overnight cultures were diluted to an optical density of $0.2,0.02,0002$, and 0.0002 at $600 \mathrm{~nm}$ by serial dilution. Five microliters of each dilution was spotted on YNB-URA agar plates which contain different amounts of boric acid. Plates were incubated at $30{ }^{\circ} \mathrm{C}$ for 3 days and then photographed. For growth curves, overnight cultures were diluted to an optical density of 0.05 for YPD at $600 \mathrm{~nm}$. The cultures were incubated in YPD media containing $50 \mathrm{mM}$ boric acid and $\mathrm{OD}_{600}$ measurements were taken every $2 \mathrm{~h}$.

\subsection{Cloning of the YMR279c and YOR378w genes}

The cloning of the YMR279c and YOR378w genes were achieved by using the Gateway Cloning System (Invitrogen). Wild type alleles of YMR279c and YOR378w genes were amplified by Long PCR Enzyme Mix (Fermentas) from yeast genomic DNA. The amplified fragments were first cloned into pDONR vector and then moved into pAG426GPD. Plasmids were confirmed with sequence 
analyses. Standard lithium acetate procedure was used for yeast transformation.

\subsection{Determination of intracellular boron concentrations}

Wild type and ymr279c $\Delta$ cells were grown to logarithmic phase and then splitted into two cultures. One group was treated with $50 \mathrm{mM}$ boric acid whereas the other group remained untreated. After $1 \mathrm{~h}$ of incubation at $30^{\circ} \mathrm{C}$, cells were harvested and washed with distilled water three times. Cell pellets were disrupted in $500 \mu$ lysis buffer ( $20 \mathrm{mM}$ Tris, pH 7.6, $100 \mathrm{mM} \mathrm{NaCl}, 1 \mathrm{mM}$ EDTA $\mathrm{pH} 8,2 \%$ Triton $\mathrm{X}-100,1 \%$ sodium dodecyl sulfate, $10 \mathrm{mg} / \mathrm{ml}$ protease inhibitor cocktail [Sigma], $500 \mu$ l glass beads) by vortexing. The intracellular boron concentrations were determined by Boron Cell Test kit (Merck) using cell extracts. Samples were normalized by cell numbers. The measurement sensitivity of the kit was $8 \times 10^{-7} \mathrm{M}(0.05 \mathrm{mg}$ boron/L) as stated by the manufacturer.

\subsection{RNA isolation and real-time PCR analyses}

Total RNA isolations from $50 \mathrm{mM}$ boric acid treated or untreated samples were performed using the PureLink RNA kit (Invitrogen) and genomic DNA contaminations were removed by DNase treatment (Fermentas). For cDNA synthesis, the First Strand cDNA synthesis kit (Fermentas) was used. ATR1, YMR279C and YOR378W genes were amplified by using Fermentas Maxima ${ }^{\mathrm{TM}}$ SYBER Green qPCR Master Mix $(2 \times)$. ACT1 gene was used as the internal control. Real-time PCR experiments were performed with an IQ5 real-time PCR system (Bio-Rad) and conditions were as follow: 40 cycles at $94{ }^{\circ} \mathrm{C}$ for $30 \mathrm{~s}, 62{ }^{\circ} \mathrm{C}$ for $30 \mathrm{~s}$, and $72{ }^{\circ} \mathrm{C}$ for $30 \mathrm{~s}$. The primers used for ATR1 amplification were ATRRT1F (5'-GGCAGAC TAGGTGACATATACG-3') and ATRRT1R (5'-TTGTTTTGGGTCCTCGGT AC-3'). The primers for YMR279c amplification were YMRRTF (5'TTTCGATGGATTGGACTGGG-3') and YMRRTR (5'-TCCAAATACCAAA CGACCCC- $\left.3^{\prime}\right)$ and those used for YOR379w were YORRTF (5'ATGGGCTTACTGGATAATGGG-3) and YORRTR (5'-AATGGTAGCAGAGGGAAAGC- $3^{\prime}$ ). Finally, the primers used for $A C T 1$ gene amplification were ACT1F (5'-ACGTTCCAGCCTTCTACGTTTCCA-3') and ACT1B (5'-ACGTGAGTAACACCATCACCGGAA-3').

\subsection{Bioinformatic analyses}

Multiple sequence alignments were performed via T-coffee 6.85 [12] software using the default parameters. Pairwise sequence alignments were performed with the Lalign 2.1.30 on-line version, and sequence identity percentages were calculated using the same software on DNA scoring matrix via default parameters [13].

\section{Results}

\subsection{Boron effects on cell growth}

Cells lacking YMR279c and YOR378w genes were previously tested for boron sensitivity and they were found to be not boron sensitive [9]. Based on this observation, both YMR279c and YOR378w genes have not been considered for further investigation

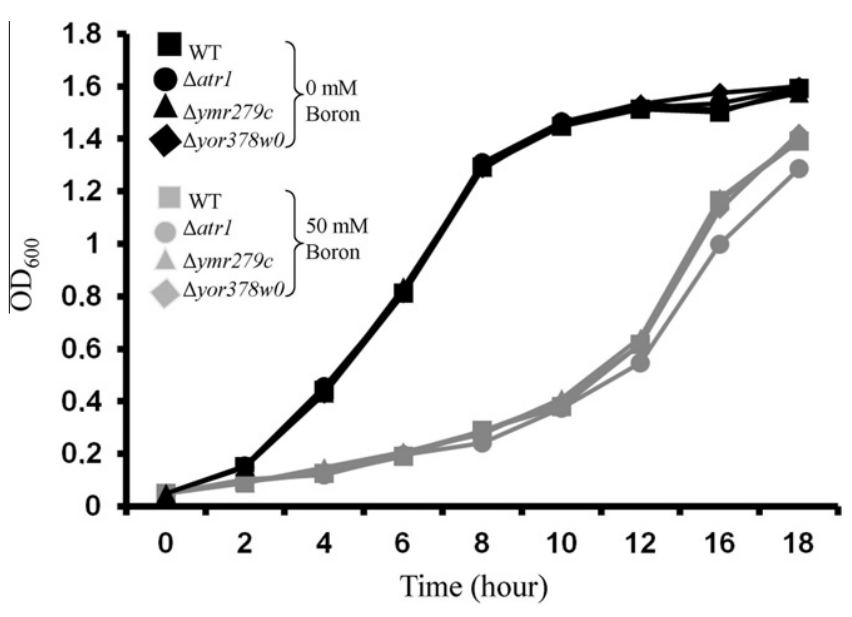

Fig. 1. Boric acid tolerance levels of atr1 $\Delta, y m r 279 c \Delta$ and yor378w $\Delta$ mutants. Overnight cultures of wild type, atr1 $\Delta, y m r 279 c \Delta$ and yor378w $\Delta$ cells were diluted to an optical density of 0.05 and incubated in the presence or absence of $50 \mathrm{mM}$ boric acid. Cell growth was followed by $\mathrm{OD}_{600}$ measurements.

for their roles in boron tolerance. However, when their primary structures are considered, Ymr279c and Yor378w show 65\% and $40 \%$ homology to Atr1, respectively. The N-terminal region is the most heterogenous part in all three proteins (Fig. S1). In spite of the similarities in their ORFs, they do not contain similar consensus sequences and are not regulated by common transcription factors (Table 1) as deducted from the Yeastract (Yeast Search for Transcriptional Regulators and Consensus Tracking) database [14]. There is only one transcription factor, Cin5, seems to regulate both ATR1 and YMR279c. Cin5 is a basic leucine zipper transcription factor that belongs to the Yap1 family and plays a role in multidrug resistance $[15,16]$. On the other hand, Met4 is the only transcription factor which is common to both ATR1 and YOR378w genes. Met4 plays a role in biosynthesis of sulfur containing amino acids [17]. Regulation of ATR1 and its paralogs by different transcription factors suggest that they may function under different stress conditions.

The lack of regulatory link between ATR1 and its paralogs prompted us to further analyze them regarding their roles in boron stress tolerance. Specifically, we wanted to know whether transcriptional regulation of YMR279c and YOR378w or sequence differences in their ORFs is responsible for boron insensitive phenotypes of ymr279c $\Delta$ and yor378w $\Delta$ mutants as noticed previously [9]. We first confirmed the results of Kaya et al. [9] by checking the growth rates of the mutants on boron containing media. Both ymr279c $\Delta$ and yor378w 4 mutants could grow well in media containing $50 \mathrm{mM}$ of boron, whereas atr1 $\Delta$ cells grew slightly slower (Fig. 1). Thus, absence of YMR279c and YOR378w genes does not sensitize cells for boron. In order to see whether overproduction of these genes from a constitutive promoter on a high copy number plasmid would help to tolerate boron, both genes were cloned and overexpressed in wild-type cells. As seen in Fig. 2 (upper panel), overexpression of YMR279c, but not YOR378w provided a significant level of boron tolerance to

Table 1

Transcription factors shown to regulate ATR1 and its paralogs.

\begin{tabular}{|c|c|}
\hline Gene name & Transcription factors \\
\hline ATR1 & Cad1p, Cin5p, Fhl1p, Fkh2p, Gcn4p, Met4p, Msn2p, Msn4p, Pdr1p, Pdr3p, Phd1p, Put3p, Rim101p, Rpn4p, Sfp1p, Stp2p, Yap1p, Yap6p, Yap7p \\
\hline YMR279C & Aft1p, Aft2p, Cat8p, Cin5p, Mal33p, Mbp1p, Rfx1p, Swi4p \\
\hline YOR378W & Adr1p, Crz1p, Gal4p, Met4p, Rap1p, Rdr1p, Rfx1p, Sok2p, Ste12p, Swi4p \\
\hline
\end{tabular}

Data were extracted from YEASTRACT database (14), using the search option for transcription factors. 


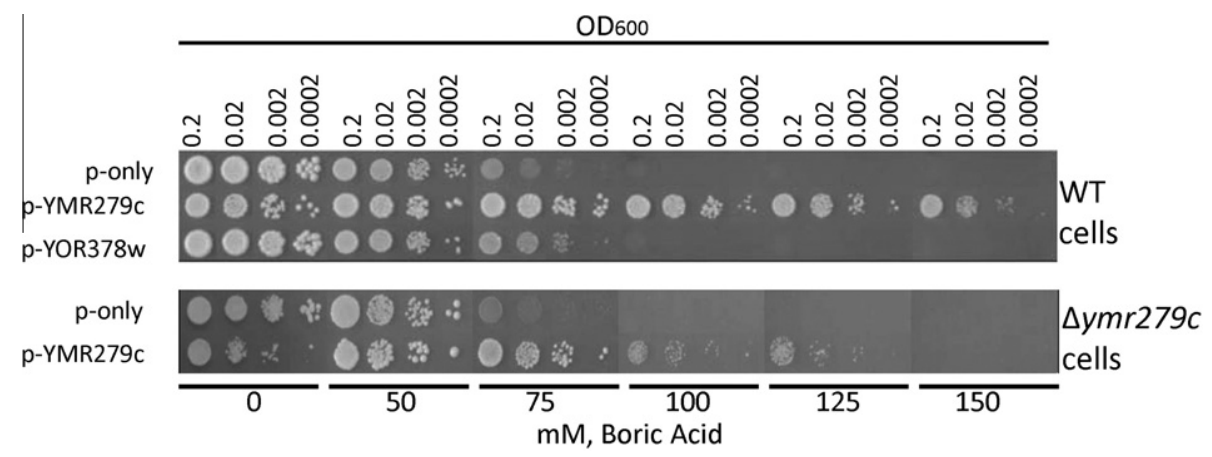

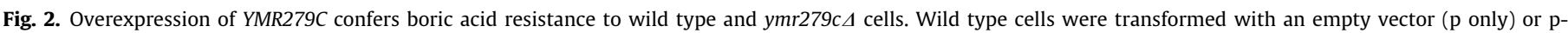

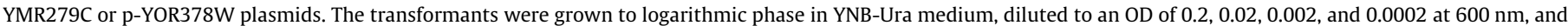

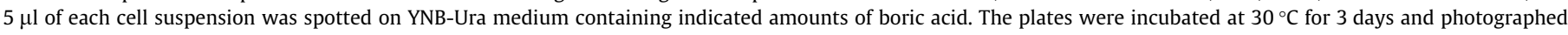

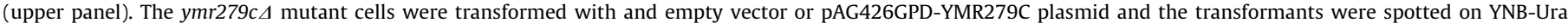
plates containing indicated amounts of boric acid and incubated at $30^{\circ} \mathrm{C}$ for 3 days (lower panel).

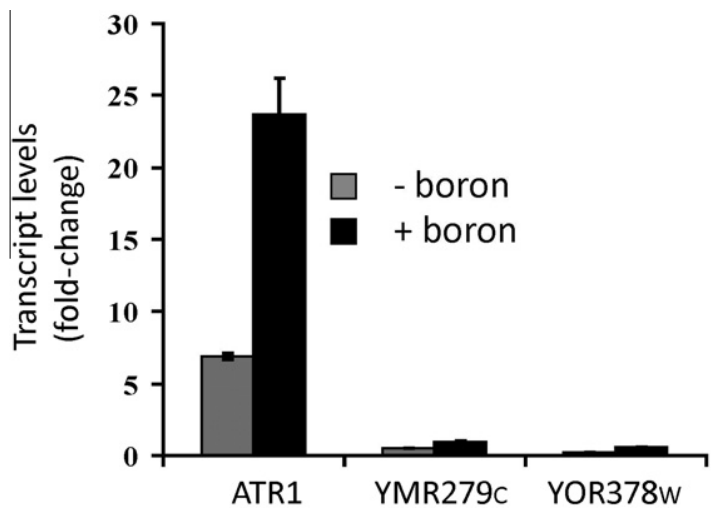

Fig. 3. Transcriptional expression profiles of $Y M R 279 C$ and $Y O R 378 W$ in response to boric acid treatment. Wild type cells were grown to logarithmic phase and were split into two groups, one of them was treated with $50 \mathrm{mM}$ boric acid for $1 \mathrm{~h}$. ATR1, YMR279C and YOR378W mRNA levels were detected by a real-time PCR analyses. $A C T 1$ was used as the internal control.

wild-type cells. YMR279c overexpression also provided boron resistance to ymr279c $\Delta$ mutants (Fig. 2, lower panel). Thus, constitutive high level expression of YMR279c helps cells to tolerate higher concentration of boron.

\subsection{Expression analyses of YMR279c and YOR378w in response to boron}

A physiological stress response gene is expected to be induced upon treatment with its stressor. Since YMR279c can function as a boron tolerance gene, we investigated whether boron stress is transduced to transcriptional machinery responsible for YMR279c transcription. To do so, cells were challenged with boron and the transcript levels of ATR1, YMR279c, and YOR378w genes were analyzed in parallel by a real-time PCR approach. As seen in Fig. 3 basal levels of YMR279c and YOR378w transcripts were 14-fold and 34-fold lower, respectively, in untreated cells when compared to that of ATR1, and they showed a less than 2-fold increase upon boron treatment. However, high basal level of ATR1 mRNA was further upregulated by 4 -fold in response to boron. This mode of ATR1 regulation was previously noticed [9]. Thus, even though Ymr279c has a role in boron detoxification, its transcription is not significantly regulated by boron stress, which suggest that Ymr279c role in boron tolerance may not be very important under native conditions.

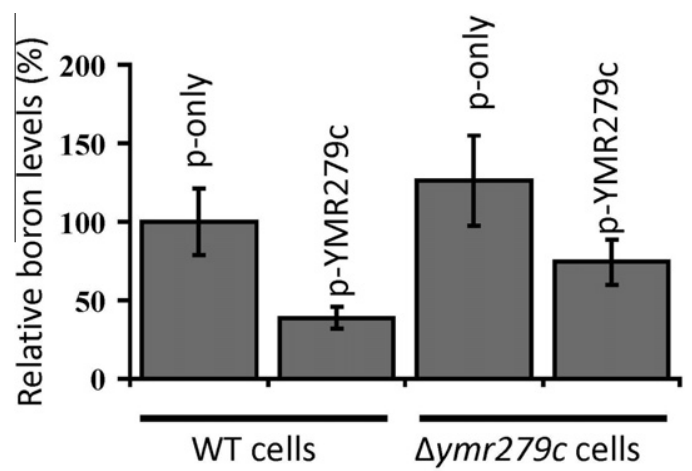

Fig. 4. Intracellular boron levels in wild-type and ymr279c $\Delta$ cells that were exposed to $50 \mathrm{mM}$ boric acid for $1 \mathrm{~h}$. Cells were transformed with either empty vector or YMR279C overexpression plasmid ( $\mathrm{p}$-YMR279C). Boron levels were normalized by total cell counts. Error bars represent three separate measurements.

\subsection{YMR279c expression lowers intracellular boron level after boron treatment}

Previously two membrane proteins, Bor1 and Atr1, were shown to provide boron resistance by reducing intracellular boron levels $[8,9]$. In order to test whether Ymr279c functions as boron efflux transporter similar to Bor1 or Atr1, intracellular boron levels of cells overexpressing YMR279c or containing sham vector were determined after boron application. As shown in Fig. 4, ymr279c $\Delta$ cells with empty plasmid had slightly higher (25\%) amount of boron when compared to that of empty plasmid carrying wild-type cells. YMR279c overexpression in both wild-type and ymr279c $\Delta$ mutants decreased cellular boron levels by $60 \%$ and $40 \%$, respectively, when compared to their corresponding sham vector carrying cells. These results suggest that YMR279c expression help cells to lower intracellular boron concentration when they are exposed to boron stress.

\section{Discussion}

In yeast, mechanisms of boron resistance are centered around exportation of boron out of cells. Two membrane proteins, Atr1 and Bor1, were previously characterized as membrane boron efflux transporters $[9,18]$. Here we showed that Ymr279c, one of the two paralogs of Atr1, provides boron resistance by decreasing intracellular boron concentrations similar to Atr1. However, there are differences between Atr1 and Ymr279c roles in boron tolerance. 
YMR279c is slightly upregulated by boron at the transcriptional level and provides boron resistance only if it is expressed ectopically from a constitutive promoter. Moreover, its overexpression leads to less boron resistance when compared to ATR1, whose expression leads to toleration of up to $220 \mathrm{mM}$ boric acid [9].

Interestingly, when Kaya et al. [9] screened a yeast gDNA library for the genes that confer resistance for boron in wild-type cells, YMR279c gene was not identified as a boron tolerance gene. A possible explanation for this outcome is that YMR279c containing cells were probably not able to grow under the experimental conditions, because they picked the transformants that could tolerate up to $200 \mathrm{mM}$ of boron. Here we showed that YMR279c overexpression helps to tolerate only about $150 \mathrm{mM}$ of boron. In a separate genome-wide screening, yeast deletion mutants that are sensitive or resistant to boron stress were identified, but ymr279c $\Delta$ mutants escaped from detection [19], because of the fact that $y m r 279 c \Delta$ cells are not boron sensitive. Thus, our special interest in testing ATR1 paralogs for boron stress response resulted in identification of YMR279c as the third boron efflux transporter in yeast.

Gene families are usually formed via gene duplication events and additional copies of same genes confer selective advantages to the host [20] by undergoing an accelerated evolution [21]. Thus, members of gene families may obtain novel functions and gain different regulatory patterns. It seems that Atr1 and its paralogs have been differentiated both function- and regulation-wise over the course of the evolution. Among them, Atr1 is the main boron exporter, Ymr279c has potential to pump boron out of cell and Yor379w has no roles in boron tolerance.

\section{Acknowledgments}

We thank to IYTE-Biotechnology center and IYTE-Biological mass spectrometry facilities for some instrumental help. This work was supported by TUBITAK $108 \mathrm{~T} 181$ and TUBA-GEBIP Grants to A.K.

\section{Appendix A. Supplementary data}

Supplementary data associated with this article can be found, in the online version, at doi:10.1016/j.bbrc.2011.05.080.

\section{References}

[1] R.I. Rowe, C.D. Eckhert, Boron is required for zebrafish embryogenesis, The Journal of Experimental Biology 202 (1999) 1649-1654.
[2] Lidefonso Bonilla, Mercedes Garcia-Gonzalez, P. Mateo, Boron requirement in cyanobacteria, Plant Physiology 94 (1990) 1554-1560.

[3] A. Bennett, R.I. Rowe, N. Soch, C.D. Eckhert, Boron stimulates yeast (Saccharomyces cerevisiae) growth, The Journal of Nutrition 0022 (1999) 2236-2238.

[4] Kyotaro Noguchi, Miho Yasumori, Takahiro Imai, Satoshi Naito, Toshiro Matsunaga, Hisao Oda, Hiroaki Hayashi, Mitsuo Chino, T. Fujiwara, Borl-1, an Arabidopsis thaliana mutant that requires a high level of boron, Plant Physiology 115 (1997) 901-906.

[5] K. Waeington, The effect of boric acid and borax on the broad bean and certain other plants, Annals of Botany XXV11 (1923) 629-672.

[6] M.A. O'Neill, S. Eberhard, P. Albersheim, A.G. Darvill, Requirement of borate cross-linking of cell wall rhamnogalacturonan II for Arabidopsis growth, Science 294 (2001) 846-849.

[7] Junpei Takano, Kyotaro Noguchi, Miho Yasumori, Masaharu Kobayashi, Zofia Gajdos, Kyoko Miwa, Hiroaki Hayashi, T. Yoneyama, T. Fujiwara, Arabidopsis boron transporter for xylem loading, Nature 420 (2002) 337-340.

[8] J. Takano, M. Kobayashi, Y. Noda, T. Fujiwara, Saccharomyces cerevisiae Bor1p is a boron exporter and a key determinant of boron tolerance, FEMS Microbiology Letters 267 (2007) 230-235.

[9] A. Kaya, H.C. Karakaya, D.E. Fomenko, V.N. Gladyshev, A. Koc, Identification of a novel system for boron transport: Atr1 is a main boron exporter in yeast, Molecular and Cellular Biology 29 (2009) 3665-3674.

[10] Y. Gbelska, J.J. Krijger, K.D. Breunig, Evolution of gene families: the multidrug resistance transporter genes in five related yeast species, FEMS Yeast Research 6 (2006) 345-355.

[11] I. Sa-Correia, S.C. dos Santos, M.C. Teixeira, T.R. Cabrito, N.P. Mira, Drug: $\mathrm{H}^{+}$ antiporters in chemical stress response in yeast, Trends in Microbiology 17 (2009) 22-31.

[12] D.G. CeÂdric Notredame, J. Higgins, T.-Coffee. Heringa, A novel method for fast and accurate multiple sequence alignment, Journal of Molecular Biology 302 (2000) 205-217.

[13] X.Q. Huang, W. Miller, A time-efficient linear-space local similarity algorithm, Advances in Applied Mathematics 12 (1991) 337-357.

[14] P.T. Monteiro, N.D. Mendes, M.C. Teixeira, S. d'Orey, S. Tenreiro, N.P. Mira, H. Pais, A.P. Francisco, A.M. Carvalho, A.B. Lourenco, I. Sa-Correia, A.L. Oliveira, A.T. Freitas, Yeastract-discoverer: new tools to improve the analysis of transcriptional regulatory associations in Saccharomyces cerevisiae, Nucleic Acids Research 36 (2008) D132-D136.

[15] I. Mendizabal, G. Rios, J.M. Mulet, R. Serrano, I.F. de Larrinoa, Yeast putative transcription factors involved in salt tolerance, FEBS Letters 425 (1998) 323328.

[16] T. Furuchi, H. Ishikawa, N. Miura, M. Ishizuka, K. Kajiya, S. Kuge, A. Naganuma, Two nuclear proteins, Cin5 and Ydr259c, confer resistance to cisplatin in Saccharomyces cerevisiae, Molecular Pharmacology 59 (2001) 470-474.

[17] P.L. Blaiseau, D. Thomas, Multiple transcriptional activation complexes tether the yeast activator Met4 to DNA, The EMBO Journal 17 (1998) 6327-6336.

[18] M.L. Jennings, T.R. Howren, J. Cui, M. Winters, R. Hannigan, Transport and regulatory characteristics of the yeast bicarbonate transporter homolog Bor1p, American Journal of Physiology. Cell Physiology 293 (2007) C468-476.

[19] I. Uluisik, A. Kaya, E.S. Unlu, K. Avsar, H.C. Karakaya, T. Yalcin, A. Koc, Genomewide identification of genes that play a role in boron stress response in yeast, Genomics 97 (2011) 106-111.

[20] Martin A. Nowak, Maarten C. Boerlijst, J. Cooke, J.M. Smith, Evolution of genetic redundancy, Nature 388 (1997) 167-171.

[21] Manolis Kellis, B.W. Birren, E.S. Lander, Proof and evolutionary analysis of ancient genome duplication in the yeast Saccharomyces cerevisiae, Nature 428 (2004) 617-624. 